\title{
Cardiac events occurred commonly among apparently healthy Filipinos with the Brugada ECG pattern in the LIFECARE cohort
}

\author{
Giselle G Gervacio, ${ }^{1}$ Jaime Alfonso Manalo Aherrera, ${ }^{1}$ Rody G Sy, ${ }_{1}$ Lauro L Abrahan IV, ${ }^{1}$ \\ Michael Joseph Agbayani, ${ }^{1}$ Felix Eduardo Punzalan, ${ }^{1}$ Elmer Jasper B Llanes, ${ }^{1}$ \\ Paul Ferdinand M Reganit, ${ }_{1}^{1}$ Olivia T Sison, ${ }^{2}$ E Shyong Tai, ${ }^{3}$ Felicidad V Velandria, ${ }^{2}$ \\ Allan Gumatay, ${ }^{4}$ Nina T Castillo-Carandang ${ }^{2}$
}

${ }^{1}$ Department of Medicine, University of the Philippines Philippine General Hospital, Manila, Philippines

${ }^{2}$ Department of Clinical Epidemiology, University of the Philippines, Manila, Philippines ${ }^{3}$ Saw Swee Hock School of

Public Health and Department of Medicine Yong Loo Lin School of Medicine, National University Health Care System Singapore, Singapore

${ }^{4}$ Manila Doctors Hospital, Manila, Philippines

Correspondence to Dr Giselle G Gervacio, Department of Medicine, University of the PhilippinesPhilippine General Hospital, Manila 1000, Philippines; giselle_gervacio@yahoo.com

Received 7 January 2018 Revised 18 April 2018 Accepted 19 April 2018
Check for updates

To cite: Gervacio GG, Aherrera JAM, Sy RG, et al. Heart Asia 2018:10:e010969. doi:10.1136/ heartasia-2017-010969

\section{ABSTRACT}

Background Brugada syndrome is the mechanism for sudden unexplained death. The Brugada ECG pattern is found in $2 \%$ of Filipinos. There is a knowledge gap on the clinical outcome of these individuals. The clinical profile and 5-year cardiac event rate of individuals with the Brugada ECG pattern were determined in this cohort. Methods This is a sub-study of LIFECARE (Life Course Study in Cardiovascular Disease Epidemiology), a community based cohort enrolling healthy individuals 20 to 50 years old conducted in 2009-2010. ECGs of all enrollees were screened independently by three cardiologists. The prevalence of the coved Brugada ECG pattern was ascertained, and the 5-year cardiac event rate was determined among those individuals with this pattern. The participants were contacted to determine the occurrence of cardiac events, which included syncope, presyncope, seizures, cardiac arrest and unexplained vehicular accidents.

Results A total of 3072 ECGs were reviewed, and 14 subjects $(0.4 \%)$ with the coved Brugada ECG pattern were identified. Four had a cardiac event on follow-up at 5 years, but all remained alive. Most of these 14 coved Brugada individuals were healthy and asymptomatic at baseline.

Conclusion Cardiac events occurred commonly among initially asymptomatic Filipinos with the coved Brugada ECG pattern. Such patients need to be followed up closely.

\section{INTRODUCTION}

Sudden unexplained death syndrome (SUDS) is the proposed explanation for bangungut. Bangungut was first described in 1917 in the Philippines, and refers to a peculiar pattern of death found among previously healthy young males. It is called lai tai in Thailand and pokkuri in Japan. Bangungut occurs in $0.043 \%$ of Filipinos. It is more prevalent in Southeast Asia, where $70 \%$ of sudden deaths are unexplained. ${ }^{1}$

The Brugada ECG pattern, characterised by $\mathrm{J}$ point and ST segment elevation in the right precordial leads, is the most useful clinical marker of SUDS. It is most useful when found among survivors of sudden death and among those with previous reports of syncope or pre-syncope. It is less useful if found in those who are asymptomatic. ${ }^{2}$ Conflicting reports exist on their prognosis. Initial reports from Brugada's group reported a 14\% event rate. ${ }^{3}$ However, this was not confirmed by Priori and other groups. ${ }^{4}$ Our group is focusing on apparently healthy Brugada ECG individuals identified in the community. In a previous study, we followed seven subjects with Brugada type 1 pattern identified during a nationwide survey in 2003 and found that of these seven initially asymptomatic individuals, three had developed cardiac events 4 years after initial diagnosis.

The objective of this study was to determine the prevalence, profile and prognosis of apparently healthy individuals with the Brugada pattern (type 1) on ECG from LIFECARE (Life Course Study in Cardiovascular Disease Epidemiology), a Filipino community-based prospective cohort of apparently healthy individuals aged 20 to 50 years old enrolled from 2009 to 2010 and subsequently followed in 2015-2016. Specifically, we were interested in determining the rate of sudden unexplained death, syncope, presyncope, seizure and unexplained accidents at 6 years after initial diagnosis in this group of individuals. This definition of possible arrhythmic events was taken from a large cohort of Japanese subjects with the Brugada ECG pattern. ${ }^{6}$ Accumulation of more data on prognosis will help fill the knowledge gap on the natural course of asymptomatic individuals in the community with the Brugada ECG sign.

\section{SUBJECTS AND METHODS General procedure}

A total of 3072 (1329 males) young healthy individuals aged 20-50 years were enrolled from four major provinces in the Philippines as part of the LIFECARE cohort. In brief, the cohort was used to determine the prevalence of common cardiovascular risk factors. All subjects underwent a 12-lead ECG on enrolment. At 5 years, vital status was ascertained. A questionnaire to screen for the occurrence of sudden unexplained death or symptoms/circumstances suggestive of a cardiac event was administered.

\section{The LIFECARE cohort}

The Life Course Study in Cardiovascular Disease Epidemiology is a prospective cohort study that included apparently healthy participants aged 20-50 years old from rural and urban communities in Metro Manila and four provinces in Luzon 
(Rizal, Batangas, Bulacan, and Quezon). It aims to look into the effects of socioeconomic factors, psychosocial stress and lifestyle factors on the development of cardiovascular disease. Those with existing myocardial infarction, stroke, peripheral arterial disease and malignancies were excluded. Study villages in the Philippines were chosen based on accessibility and presence of support from the local government units and local collaborators. From each chosen village, household lists were obtained. Random selection of households was done using systematic random sampling. A list of eligible members per household was generated. The Kish method was then utilised to recruit only one member per household to ensure a balanced distribution of age and sex of participants. A trained field team conducted face-to-face interviews to obtain data. Twelve-lead ECGs were taken from all included participants, as well as blood pressures, fasting blood sugar and lipid levels, and serum creatinine. The above mentioned baseline data collection was done from 2009 to 2010. A more detailed description of the protocol is published elsewhere. ${ }^{7}$

\section{Definition of the Brugada ECG pattern}

We used the 2015 criteria for the diagnosis of the Brugada ECG pattern. This includes a $\geq 2 \mathrm{~mm} \mathrm{~J}$ point elevation followed by $\mathrm{a} \geq 1 \mathrm{~mm}$ coved type of ST elevation and T wave inversion in $\geq 1$ of the right precordial leads V1 to V3. ${ }^{8}$ A saddleback pattern was not considered diagnostic of Brugada unless it was accompanied by conversion to the coved pattern. ${ }^{9}$ Those with the Brugada type 2 pattern (saddleback) were also identified, but being a community-based study, drug challenge to unmask the type 1 pattern was not performed.

\section{Screening of ECGs}

All 3072 resting 12-lead ECG recordings in the LIFECARE cohort done from 2009 to 2010 were screened independently by three cardiologists (one general cardiologist and two electrophysiologists) who were oriented on the definition of the Brugada ECG pattern. They were all blinded to the clinical status of the participants. The final list of participants with Brugada type 1 ECG pattern was produced after adjudication of the ECG readings by the three cardiologists. The data of these patients were reviewed, as recorded in the LIFECARE cohort. Data gathered included age, sex, place of residence, civil status, educational achievement, employment status, household salary, recent hospitalisation, smoking and alcohol consumption, blood chemistry profile (fasting blood sugar, creatinine, and lipid profile), anthropometric measurements, vital signs, past medical history, family medical history, drugs/medications being taken, and the magnitude of J point elevation and ST elevation. The characteristics and demographics of the patients identified to have the Brugada ECG pattern were compared with the rest of the cohort.

The ECGs of patients with the Brugada type 1 pattern were also examined for features considered to be high risk for malignant arrhythmias, including QRS duration $>120 \mathrm{~ms}$ in lead $\mathrm{V} 2,{ }^{10}$ corrected $\mathrm{QT}\left(\mathrm{QT}_{\mathrm{C}}\right)$ interval length $>460 \mathrm{~ms}$ in lead $\mathrm{V} 2,{ }^{11}$ fragmented QRS, ${ }^{10}{ }^{11}$ significant $\mathrm{S}$ wave $(\geq 0.1 \mathrm{mV}$ and/ or $\geq 40 \mathrm{~ms}$ ) in lead I, ${ }^{12}$ and the aVR sign ( $\mathrm{R}$ wave $\geq 3 \mathrm{~mm}$ or $\mathrm{R} / \mathrm{q}$ ratio $\geq 0.75$ in lead aVR). ${ }^{1013}$

\section{Definition of sudden unexplained death and cardiac events}

Sudden unexplained death was defined as death within 1 hour of the onset of symptoms in someone who was apparently healthy. Cardiac events included syncope, presyncope, seizures, cardiac arrest and unexplained vehicular accidents. This definition was taken from a four-decade study by Matsuo. ${ }^{6}$

The vital status was ascertained in 2015 (5-6 years after the initial ECG) by follow-up visit for all 3072 participants in the study. During this first follow-up, all 14 participants with the Brugada pattern were alive. In 2016, a second follow-up was done by face-to-face interview to determine the occurrence of cardiac events among those with the Brugada type 1 pattern. During this second round, only 10 of the 14 participants were located and consented for another interview. Adjudication was performed and for syncope, effort was made to differentiate arrhythmic syncope from vasovagal syncope. Discussions were held to determine if symptoms could be attributed to a spontaneously terminating ventricular arrhythmia. Further clarifications were allowed via telephone contact. Electrophysiologic and genetic testing were not performed.

In summary, the following data were collected:

1. Prevalence of the Brugada ECG coved pattern in the LIFECARE cohort.

2. Incidence of sudden unexplained death among the Brugada ECG population (and subpopulation).

3. Incidence of cardiac events (syncope, presyncope, sudden death, seizures and unexplained accidents) among the Brugada ECG population.

4. Percentage of the group that developed cardiac events that were initially asymptomatic.

5. Percentage of the group with cardiac events that had a first degree relative with sudden cardiac death.

\section{Statistical analysis}

From the 3072 ECGs, we extracted those with the coved type of Brugada ECG pattern and calculated the prevalence at the time of examination. The data collected were entered using CSPro 4.1 and analysed using Stata version 12.0 (StataCorp LP1, College Station, Texas, USA). Descriptive statistics such as mean and SD were calculated for continuous variables and by percentages for categorical variables. Pearson's $\chi^{2}$ tests and Fisher's exact tests were used to determine group differences in Brugada type pattern. An $\alpha$ of 0.05 was used to determine statistical significance.

\section{RESULTS}

Of the 3072 participants of the LIFECARE cohort, 14 had the coved type (0.4\%). Among those with the Brugada type 1 pattern, the average age of the participants was 34 years and the majority belonged to the 40-50 years age group, were males, lived in an urban area, married, non-college graduates, employed, and current alcohol beverage drinkers. Likewise, there were more males and married participants in the Brugada group ( $\mathrm{P}$ value 0.001 and 0.024 , respectively). Table 1 summarises the characteristics of these 14 participants, compared with the other individuals in the cohort. There were significantly more males and single/widowed/separated individuals in the Brugada type 1 group versus those with no Brugada pattern. The Brugada type 2 pattern was seen in 119 (3.9\%) participants.

Standard cardiovascular risk factors were not significantly different between the two groups, with a mean systolic blood pressure of $128 \mathrm{~mm} \mathrm{Hg}$ and mean body mass index of $21 \mathrm{~kg} /$ $\mathrm{m}^{2}$. The biochemical profile of the Brugada type 1 group was normal and not different between the two groups. All had a fasting blood sugar $<126 \mathrm{mg} / \mathrm{dL}$ and serum creatinine $<126$ $\mu \mathrm{mol} / \mathrm{L}$. Most had a low-density lipoprotein (LDL) concentration $<160 \mathrm{mg} / \mathrm{dL}$. See table 2 for further details. 
Table 1 Sociodemographic profile of the Philippine LIFECARE cohort $(n=3072)$

\begin{tabular}{lccc}
\hline Variable & $\begin{array}{l}\text { With Brugada } \\
\text { type 1 pattern } \\
(\mathbf{n}=14)\end{array}$ & $\begin{array}{l}\text { No Brugada } \\
\text { type 1 pattern } \\
(\mathbf{n}=3058)\end{array}$ & P value \\
\hline Age group, $\mathrm{n}(\mathrm{col} \%)$ & & & \\
\hline 20-29 years & $5(35.7)$ & $856(28.0)$ & $0.072^{*}$ \\
\hline 30-39 years & $1(7.1)$ & $1101(36.0)$ & \\
\hline 40-50 years & $8(57.1)$ & $1101(36.0)$ & \\
Males & $12(85.7)$ & $1316(43.0)$ & $0.001^{*}$ \\
\hline Urban place of residence & $8(57.1)$ & $1538(50.3)$ & $0.544^{*}$ \\
Married or with a live-in partner & $7(50.0)$ & $2363(77.3)$ & $0.024 \dagger$ \\
\hline Attained a college degree & $4(28.6)$ & $796(26.0)$ & $0.879 \dagger$ \\
Employed & $11(78.6)$ & $2058(67.3)$ & $0.569 \dagger$ \\
Current or previous smoker & $7(50.0)$ & $1057(34.6)$ & $0.234 \dagger$ \\
Current alcohol drinker & $11(78.6)$ & $1801(58.9)$ & $0.135^{*}$ \\
\hline
\end{tabular}

${ }^{*}$ Group comparisons using $\chi^{2}$ test.

tGroup comparisons using Fisher's exact test.

\section{Occurrence of cardiac events}

Of the 14 participants with the coved type Brugada ECG, all were alive at the time of a vital status check in $2015(0 \%$ mortality in 5 years). In 2016, we are able to interview 10 of the 14 for the occurrence of cardiac events. The other four could not be located despite an exhaustive search during the second round, which included site visits, telephone calls, social media and/or email.

Four of the 10 participants who were followed-up developed a cardiac event within 5 years. These were adjudicated to be cardiac events and consisted of a middle-aged participant with palpitations and severe dizziness; a young participant with presyncope, described as a feeling of 'almost blacking out'; a middle-aged participant with presyncope and an unexplained accident at work which may have been from presyncope; and a middle-aged participant who developed frank syncope (table 3 ).

Thirteen of the 14 participants with the Brugada type 1 pattern were asymptomatic before recruitment. The sole individual who had symptoms before recruitment was the fourth participant (see table 3). The participant had been experiencing occasional palpitations for the past 7 years.

Of the 14 participants with Brugada type 1 pattern, only one had a first-degree relative who had sudden cardiac death during sleep at a young age. Another participant (who had a cardiac event) had a cousin who had sudden cardiac death during sleep. The other participants had no family history of sudden cardiac death.

Table 2 Blood pressure and body mass index of the LIFECARE cohort $(n=3072)$

\begin{tabular}{|c|c|c|c|}
\hline Variable & $\begin{array}{l}\text { With Brugada type } \\
1 \text { pattern }(n=14)\end{array}$ & $\begin{array}{l}\text { No Brugada type } 1 \\
\text { pattern }(n=3058)\end{array}$ & $P$ value \\
\hline \multicolumn{4}{|l|}{ Systolic blood pressure } \\
\hline $\mathrm{n}$ & 14 & 3056 & \\
\hline Mean (SD) (mm Hg) & $128.1(17.2)$ & $121.2(18.5)$ & $0.112^{*}$ \\
\hline \multicolumn{4}{|l|}{ Diastolic blood pressure } \\
\hline $\mathrm{n}$ & 14 & 3056 & \\
\hline Mean (SD) (mm Hg) & $72.7(11.5)$ & $72.4(12.0)$ & $0.802^{*}$ \\
\hline \multicolumn{4}{|l|}{ Body mass index } \\
\hline $\mathrm{n}$ & 14 & 3058 & \\
\hline Mean (SD) $\left(\mathrm{kg} / \mathrm{m}^{2}\right)$ & $21.0(2.8)$ & $23.4(4.3)$ & $0.045^{*}$ \\
\hline
\end{tabular}

Table 3 Description of participants with Brugada pattern

\begin{tabular}{|c|c|c|c|c|}
\hline & $\begin{array}{l}\text { At } \\
5 \text { years }\end{array}$ & Cardiac events & $\begin{array}{l}\text { Family history } \\
\text { (first degree } \\
\text { relative) of } \\
\text { sudden death }\end{array}$ & $\begin{array}{l}\text { Previously } \\
\text { asymptomatic }\end{array}$ \\
\hline 1 & $\begin{array}{l}\text { Alive by } \\
5 \text { years }\end{array}$ & $\begin{array}{l}\text { Lost to follow-up (on the sixth } \\
\text { year) }\end{array}$ & None & Yes \\
\hline 2 & Alive & None & None & Yes \\
\hline 3 & Alive & $\begin{array}{l}\text { Presyncope, occasional } \\
\text { palpitations associated with } \\
\text { severe dizziness }\end{array}$ & None & No \\
\hline 4 & Alive & None & None & Yes \\
\hline 5 & Alive & None & $\begin{array}{l}\text { Yes (sibling } \\
\text { had sudden } \\
\text { death during } \\
\text { sleep at a } \\
\text { young age) }\end{array}$ & Yes \\
\hline 6 & Alive & None & None & Yes \\
\hline 7 & Alive & $\begin{array}{l}\text { Presyncope, turned cyanotic, } \\
\text { during exertion (working in } \\
\text { a truck) }\end{array}$ & None & Yes \\
\hline 8 & Alive & $\begin{array}{l}\text { Presyncope/syncope, accident } \\
\text { during work }\end{array}$ & None & Yes \\
\hline 9 & $\begin{array}{l}\text { Alive by } \\
5 \text { years }\end{array}$ & $\begin{array}{l}\text { Lost to follow-up (on the sixth } \\
\text { year) }\end{array}$ & None & Yes \\
\hline 10 & $\begin{array}{l}\text { Alive by } \\
5 \text { years }\end{array}$ & $\begin{array}{l}\text { Lost to follow-up (on the sixth } \\
\text { year) }\end{array}$ & None & Yes \\
\hline 11 & $\begin{array}{l}\text { Alive by } \\
5 \text { years }\end{array}$ & $\begin{array}{l}\text { Lost to follow-up (on the sixth } \\
\text { year) }\end{array}$ & None & Yes \\
\hline 12 & Alive & None & None & Yes \\
\hline 13 & Alive & None & None & Yes \\
\hline 14 & Alive & Syncope after exertion & None & Yes \\
\hline
\end{tabular}

Table 4 shows the high-risk ECG features that were examined in those with the Brugada type 1 pattern. The most common high-risk feature among the 14 individuals was the presence of a fragmented QRS (9 out of 14,64\%), followed by a prominent $\mathrm{S}$ wave in lead I (7 out of 14, 50\%). None of the 14 participants had a prolonged $\mathrm{QT}_{\mathrm{C}}$ interval lasting more than $460 \mathrm{~ms}$.

\section{DISCUSSION}

Sudden unexplained death, first described in the Philippines in 1917, occurs more commonly in Southeast Asia. In the Philippines $^{14}$ and Thailand the incidence appears to be around 40 per 100000 compared with Europe where it is a mere 1 per 100000 . Its marker, the Brugada ECG pattern, is found rarely in the USA $(0.02 \%$ for type $1 \%$ and $0.03 \%$ for types 2 and 3$)$, whereas it is found more commonly in Asia (0-0.36\% for type 1 and 0.12 $2.23 \%$ for types 2 and 3). There are more Brugada types 2 and 3 in the Philippines and Asia (1.8-2.5\% and 0.12-2.23\%, respectively) compared with the USA and Europe $(0.03 \%$ and $1.5 \%$, respectively). ${ }^{5}$ A meta-analysis including 24 studies estimated the worldwide prevalence of the Brugada ECG pattern to be $0.4 \%$, with a prevalence of $0.9 \%$ in Asia.$^{15}$

\section{Data on apparently healthy Filipinos: prevalence and demographics}

Our community based study of 5 years confirmed that the prevalence of the Brugada coved pattern is higher in the Philippines than in Western countries.

In our cohort, the typical Filipino with the Brugada pattern is a male in his 40 s from a rural area who is single/widowed and employed. This profile is consistent with the average 
Table 4 High-risk ECG features of participants with Brugada pattern

\begin{tabular}{llllll}
\hline & $\begin{array}{l}\text { QRS duration* } \\
\text { (ms) }\end{array}$ & $\begin{array}{l}\text { QT }_{c} \text { interval* }^{*} \\
\text { (ms) }\end{array}$ & $\begin{array}{l}\text { Fragmented } \\
\text { QRS }\end{array}$ & $\begin{array}{l}\text { S wave in } \\
\text { lead I }\end{array}$ & aVR sign \\
\hline 1 & 120 & 340 & No & Yes & Yes \\
\hline 2 & 100 & 416 & Yes & No & No \\
$3 \dagger$ & 80 & 420 & Yes & No & No \\
\hline 4 & 100 & 397 & No & No & No \\
\hline 5 & 120 & 373 & No & Yes & No \\
\hline 6 & 120 & 400 & No & No & Yes \\
$7 \dagger$ & 120 & 400 & Yes & Yes & No \\
$8 \dagger$ & 120 & 353 & Yes & No & No \\
\hline 9 & 120 & 340 & Yes & Yes & Yes \\
10 & 100 & 380 & Yes & No & No \\
11 & 120 & 365 & Yes & Yes & No \\
12 & 140 & 360 & Yes & Yes & No \\
13 & 120 & 400 & Yes & Yes & Yes \\
$14 \dagger$ & 140 & 320 & No & No & No \\
\hline
\end{tabular}

* Measured at lead V2

tThese participants $(3,7,8$ and 14$)$ had cardiac events.

breadwinner in a Filipino family. Since this cohort is focused on young healthy Filipinos, the majority of our participants have normal blood pressures and acceptable biochemical profiles (fasting blood sugar, lipid profile and serum creatinine).

Most of our participants are also current cigarette smokers and alcoholic beverage drinkers. These data are pertinent because a common trigger for sudden death is alcohol consumption, although the difference did not reach statistical significance. Alcohol consumption may be easily overlooked as a precipitant of the Brugada syndrome in at-risk individuals and only a few case reports report this as a trigger. ${ }^{16}$ Theoretically, alcohol intoxication is thought to have proarrhythmic effects by inhibiting sodium-channel gating, mimicking the actions of sodium-channel inhibitors. ${ }^{17}$ Sodium-channel blockers (eg, ajmaline) are standard agents to unmask Brugada syndrome. Heavy alcohol intoxication may also increase basal body temperature particularly during heat stroke, which like fever is one of the known triggers of the Brugada syndrome. ${ }^{18}$

Another finding is that the majority of these participants live in rural areas and are from lower socioeconomic groups. Hence their households have no air-conditioning. With the concurrence of global warming in a tropical country and most especially in cramped quarters, excessive body heat or elevated core temperatures may be anticipated in these participants. 'Fever-induced Brugada' is a recognised clinical entity; however, its prevalence has not been systematically evaluated. ${ }^{19}$

One study evaluated the presence of the Brugada pattern among patients with fever and found that the type I pattern is more common among such patients, suggesting that the asymptomatic pattern is more prevalent than previously estimated. ${ }^{20}$

\section{Prognosis of Brugada}

The highest risk of sudden death in Brugada syndrome is found among sudden death survivors. This is followed by symptomatic Brugada patients presenting with syncope, presyncope, seizure and unexplained accidents. In general, asymptomatic Brugada ECG carriers have a low event rate. ${ }^{4}$

Brugada et al reported a 14\% occurrence of sudden death in 28 months. ${ }^{21}$ Referral bias was likely, brought about by sicker patients, with possibly a higher incidence of affected family members initially seen at referral centres. Even more than referral bias, there is founders' bias observed among centres first describing a clinical condition. This theory seems especially possible as this finding appears not to be confirmed by other groups $^{22}$ such as Priori et al, who reported less than $0.5-1 \%$ risk of sudden death per year among asymptomatic individuals with the Brugada ECG pattern. ${ }^{23}$

Our series reflects a need to re-examine the issue of prognosis of asymptomatic Brugada. In our previous study of subjects with the coved type Brugada identified from a randomly selected sample from the general population, seven out of 3907 had the Brugada coved pattern. Three of these seven previously asymptomatic individuals developed cardiac events in 6 years. ${ }^{5}$ Our current cohort confirms that cardiac events may not be rare even among asymptomatic subjects with the Brugada coved type pattern. Our cardiac event rate of four events out of 14 patients gives an annualised incidence of $4.2 \%$, certainly higher than the $0.5-1 \%$ annualised event rate reported by Probst et al, ${ }^{2}$ Priori et $a l,{ }^{4}$ Giusteto et $a l,{ }^{22}$ Sieira $e t a l^{23}$ and Sakabe et al. ${ }^{24}$ All these studies involved Western populations with the exception of Sakabe et al, which followed Japanese subjects during periodic health examinations.

The circumstances surrounding the cardiac event in the four patients who developed events were highly suggestive of an arrhythmic event because of its occurrence in young healthy individuals, the severity of the dizziness and, in one case, the existence of a positive family history of sudden unexplained death. Participants were all carefully queried to differentiate arrhythmic syncope from other causes of syncope such as vasovagal syncope.

The deviant pattern may reflect a distinct epidemiology, perhaps due to variable genetics in Asia or from different environmental conditions such as warmer temperatures and more humid and cramped living conditions. The lack of air-conditioning in the homes of lower socioeconomic s may result in warmer conditions during sleep. The increased temperature in these places may then affect phenotypic expression, in the same way that $40^{\circ}$ fevers appear to unmask Brugada. ${ }^{25}$

Other factors that appear to increase risk include age (fourth to fifth decade, with risk declining beyond age 60), fever or hyperthermia as discussed, family history, and occurrence of the changes in the inferolateral leads, or what may be labelled as high-risk early repolarisation changes. ${ }^{26}$ There was a high prevalence of risk ECG features among our 14 patients, indicating perhaps a higher risk population compared with other studies. $^{10-13}$

Data from high-prevalence areas, particularly in Southeast Asia, should be pooled with other data in the region to determine the need to change treatment guidelines. To this end, ECG readers need to be trained to recognise the pattern, especially the type 1 pattern, which is the most specific for sudden death. While an ICD cannot be recommended for asymptomatic patients at this point, further workup may be beneficial, as well as advice on avoidance of fever, hyperthermia and alcohol binges. The use of pharmacologic agents like quinidine and cilostazol, which have been shown in studies to decrease the occurrence of arrhythmias and arrhythmic death, may present a less aggressive treatment option that may be tried in asymptomatic patients.

\section{Limitations of the study}

The population included in the study has its characteristics set by the inclusion criteria of the LIFECARE cohort, which may not be applicable to the entire Filipino community. Longer follow-up is needed to provide data on the natural course of apparently healthy individuals with the Brugada pattern on ECG. Since this is a community-based study, the major limitation is that there is 
no actual documentation of life-threatening arrhythmias, and the occurrence of potential cardiac events is deduced from a symptom-based questionnaire. However, current guidelines include arrhythmic syncope with the Brugada ECG pattern among the diagnosis of Brugada syndrome. ${ }^{27}$

The total mortality rate of the LIFECARE cohort as of 2015 is $32 / 3072$, and none of these mortalities had a Brugada type 1 pattern. Other than the 14 participants with the Brugada type 1 pattern, the remaining participants from the LIFECARE cohort were not interviewed/questioned regarding the occurrence of cardiac events (as it was not part of our objectives).

Furthermore, it has been shown that the Brugada pattern on ECG may be dynamic (ie, may change from one pattern to another, or may even normalise). We plan to conduct another study to address ECG conversion of the Brugada pattern. Early repolarisation patterns will be also studied in this same cohort of individuals.

\section{CONCLUSION}

We conclude that asymptomatic and presumably healthy individuals with the Brugada type 1 ECG pattern have a considerable event rate ( $4 \%$ per year), which appears to be higher than that reported in other series. Surveillance of these asymptomatic cases is recommended.

Key messages

What is already known about this subject?

- Brugada syndrome is the mechanism behind sudden unexplained death syndrome.

- Patients with aborted sudden death, syncope or documented episodes of ventricular tachycardia/ventricular fibrillation are at highest risk of a repeat event.

- The coved type Brugada ECG marker is found in $0.2 \%$ of the general population in the Philippines, with the majority being asymptomatic.

- In the West, the prognosis of asymptomatic Brugada is good with only $0.5-1 \%$ cardiac events annually.

What does this study add?

- In Southeast Asia, the cardiac event rate appears higher.

- In a previous study, we followed seven asymptomatic coved Brugada subjects identified from the general population, and observed that three of the seven had developed cardiac events over the span of 4 years.

- In this present study, we confirmed that the cardiac event rate is considerable among healthy individuals in the community with the Brugada type 1 ECG pattern; $21 \%$ of asymptomatic subjects developed cardiac events over 4 years, equivalent to an annualised event rate of $4.2 \%$.

How might this impact on clinical practice?

- Individuals with the coved Brugada ECG pattern need to be worked up to rule out structural heart disease.

- The occurrence of subtle symptoms such as dizziness, blurring of vision, palpitations and unexplained falls or accidents needs to be elicited, as well as a family history of sudden death.

- In such cases, defibrillator implantation must be seriously considered; avoidance of fever, hyperthermia and heavy alcohol intoxication may also be advised.
Acknowledgements The authors are grateful for the assistance from our staff (Ms Rona May de Vera and Ms Alma Amparo) and the field team for their contribution in making this project a reality. Finally, we also acknowledge the participation of another member of the LIFECARE team: Paulette D Nacpil-Dominguez, MD.

Contributors All authors contributed to the planning, conduct, and reporting of the work.

Funding Main funding for the substudy was covered by a grant from the Philippine College of Physicians. The following organiSations provided financial assistance to the project: Pfizer Asia, Pfizer International, Pfizer Philippines, Department of Health (Philippines), Philippine Council for Health and Research Development, Diabetes Philippines, Philippine Society of Hypertension, and the Philippine Lipid and Atherosclerosis Society. The University of the Philippines Manila (through the College of Medicine) and the National Institutes of Health's Institute of Clinical Epidemiology have also supported the project.

Competing interests None declared.

Patient consent Not required.

Ethics approval The protocol was submitted to the University of the Philippines Medical Research Ethics Board (UPMREB) for ethics review and approval, which conforms to the provisions of the Declaration of Helsinki. The study was approved on 12 August 2015 with a UPMREB Code (MED) 2015-310-01. The study was conducted upon approval from the UPMREB. Informed consent was taken by a research assistant during his/her personal visit from all the participants. To ensure confidentiality, the identities of patients were assigned to a number code. No other information was obtained aside from what is stated in the protocol.

Provenance and peer review Not commissioned; externally peer reviewed.

(c) Article author(s) (or their employer(s) unless otherwise stated in the text of the article) 2018. All rights reserved. No commercial use is permitted unless otherwise expressly granted.

\section{REFERENCES}

1 Gaw AC, Lee B, Gervacio-Domingo G, et al. Unraveling the enigma of Bangungut: Is sudden unexplained nocturnal death syndrome (SUNDS) in the Philippines a disease allelic to the Brugada syndrome? Philipp J Intern Med 2011;49:165-76.

2 Probst V, Veltmann C, Eckardt L, et al. Long-term prognosis of patients diagnosed with Brugada syndrome: results from the FINGER Brugada Syndrome Registry. Circulation 2010;121:635-43.

3 Brugada P, Brugada J. Right bundle branch block, persistent ST segment elevation and sudden cardiac death: a distinct clinical and electrocardiographic syndrome. A multicenter report. J Am Coll Cardiol 1992;20:1391-6.

4 Priori SG, Napolitano C, Gasparini M, et al. Natural history of Brugada syndrome: insights for risk stratification and management. Circulation 2002;105:1342-7.

5 Domingo GG, Jocson G, Dans A. Frequency of cardiac events at four years among initially asymptomatic filipinos with the Brugada type 1 electrocardiographic pattern. Am J Cardiol 2011;107:714-6.

6 Matsuo K, Akahoshi M, Nakashima E, et al. The prevalence, incidence and prognostic value of the Brugada-type electrocardiogram: a population-based study of four decades. J Am Coll Cardiol 2001;38:765-70.

7 Sy RG, Llanes EJ, Reganit PF, et al. Socio-demographic factors and the prevalence of metabolic syndrome among Filipinos from the LIFECARE cohort. J Atheroscler Thromb 2014;21(Suppl 1):S9-17.

8 Priori SG, Blomström-Lundqvist C, Mazzanti A, et al. 2015 ESC Guidelines for the management of patients with ventricular arrhythmias and the prevention of sudden cardiac death: The Task Force for the Management of Patients with Ventricular Arrhythmias and the Prevention of Sudden Cardiac Death of the European Society of Cardiology (ESC). Endorsed by: Association for European Paediatric and Congenital Cardiology (AEPC). Eur Heart J 20152015:36:2793-867.

9 Antzelevitch C, Yan GX, Ackerman MJ, et al. J-Wave syndromes expert consensus conference report: emerging concepts and gaps in knowledge. J Arrhythm 2016;32:315-39

10 Adler A, Rosso R, Chorin E, et al. Risk stratification in Brugada syndrome: clinical characteristics, electrocardiographic parameters, and auxiliary testing. Heart Rhythm 2016;13:299-310

11 Naseef A, Behr ER, Batchvarov VN. Electrocardiographic methods for diagnosis and risk stratification in the Brugada syndrome. J Saudi Heart Assoc 2015:27:96-108.

12 Calò L, Giustetto C, Martino A, et al. A new electrocardiographic marker of sudden death in Brugada syndrome: the S-wave in lead I. J Am Coll Cardiol 2016:67:1427-40.

13 Babai Bigi MA, Aslani A, Shahrzad S. aVR sign as a risk factor for lifethreatening arrhythmic events in patients with Brugada syndrome. Heart Rhythm 2007;4:1009-12.

14 Gervacio-Domingo G, Punzalan FE, Amarillo ML, et al. Sudden unexplained death during sleep occurred commonly in the general population in the Philippines: a sub study of the National Nutrition and Health Survey. J Clin Epidemiol 2007;60:567-71. 


\section{Original research}

15 Quan XQ, Li S, Liu R, et al. A meta-analytic review of prevalence for Brugada ECG patterns and the risk for death. Medicine 2016;95:e5643.

16 Ohkubo K, Nakai T, Watanabe I. Alcohol-induced ventricular fibrillation in a case of Brugada syndrome. EP Europace 2013;15:1058-5.

17 Klein G, Gardiwal A, Schaefer A, et al. Effect of ethanol on cardiac single sodium channel gating. Forensic Sci Int 2007;171:131-5.

18 Alcohol and body temperature. nutrientsreview.com (accessed on 1 Nov 2017).

19 Sharma AK, Lama L, Heist EK. Brugada pattern induced by fever. Indian Heart J 2015;67:159-60.

20 Adler A, Topaz G, Heller K, et al. Fever-induced Brugada pattern: how common is it and what does it mean? Heart Rhythm 2013;10:1375-82.

21 Brugada J, Brugada R, Antzelevitch C, et al. Long-term follow-up of individuals with the electrocardiographic pattern of right bundle-branch block and ST-segment elevation in precordial leads V1 to V3. Circulation 2002;105:73-8.

22 Giustetto C, Drago S, Demarchi PG, et al. Risk stratification of the patients with Brugada type electrocardiogram: a community-based prospective study. Europace 2009;11:507-13.
23 Sieira J, Ciconte G, Conte G, et al. Asymptomatic Brugada syndrome: clinical characterization and long-term prognosis. Circ Arrhythm Electrophysiol 2015;8:1144-50.

24 Sakabe M, Fujiki A, Tani M, et al. Proportion and prognosis of healthy people with coved or saddle-back type ST segment elevation in the right precordial leads during 10 years follow-up. Eur Heart J 2003;24:1488-93.

25 Rattanawong P, Vutthikraivit W, Charoensri A, et al. Fever-induced Brugada syndrome is more common than previously suspected: a cross-sectional study from an endemic area. Ann Noninvasive Electrocardiol 2016;21:136-41.

26 Adler A. Brugada syndrome: diagnosis, risk stratification, and management. Curr Opin Cardiol 2016;31:37-45.

27 Priori SG, Wilde AA, Horie M, et al. HRS/EHRA/APHRS expert consensus statement on the diagnosis and management of patients with inherited primary arrhythmia syndromes. Heart Rhythm 2013;10:1932-63.

28 Gervacio-Domingo GG, Daez MLO, Gloria VI LC, et al. Development of a questionnaire to measure the incidence of sudden unexplained death syndrome (bangungut) in the general population. Philipp J Cardiol 2005;33:33-7. 